\title{
Enzootic Bovine Leukosis: how to prevent the disease and control the spread of BLV infection.
}

\author{
Takeshi HAGA \\ Infection Control and Disease Prevention \\ Department of Veterinary Medical Science \\ The University of Tokyo, Tokyo, Japan \\ E-mail: ahaga@mail.ecc.u-tokyo.ac.jp
}

\begin{abstract}
Threat of animal infectious diseases, as well as that of vector-bone disease is increased in the globalized world under global warming. Enzootic Bovine Leukosis (EBL) is caused by the infection of Bovine Leukemia Virus (BLV) which belong to the family of Retiroviridae. Only a few percentages of BLV infected cattle develop fatal EBL after long incubation period from months to years. Majority of the BLV-infected animals show no apparent symptoms (asymptomatic), although they have a potential for transmissing BLV to the other cattle. BLV is mainly transmitted with the infected lymphocytes. The blood-suckling insect carrying the live blood cells from BLV-infected cattle can be a mechanical vector of BLV transmission. In contrast with acute infection, BLV can spread silently unless the proper control measure is conducted. Surveillance is critical for understanding BLV positivity, and suitable management plan of $\mathrm{BLV}$-infection for the region is to be carried out for sustainable development of livestock industries.
\end{abstract}

Keywords-EBL; BLV; Asymptomtic, blood-suckiling insect; management plan

Animal infectious diseases make an impact on the society and food security in a globalized world. Along with Gross Domestic Production (GDP), meat consumption increased in many countries [1]. Production of animal protein, and trade of meat also increased. The scale of livestock production becomes larger. In addition, global warming affects distribution of bloodsuckling insects, which may transmit infectious diseases. More than $20 \%$ of livestock product in the world is lost by animal diseases. Threat of animal infectious diseases, as well as that of vector-bone disease is increased.

Enzootic Bovine Leukosis (EBL) is one of fatal bovine infectious diseases cause by Bovine Leukemia Virus (BLV), which belongs to a family of Retroviridae. In contrast with acute disease whose onset is sudden in the order of several days after the infection of the pathogen, the appearance of EBL is very slow, which takes months to years after BLV infection. In addition, the majority of BLV-infected animals shows no clinical symptoms. A proportion $(\sim 30 \%)$ of adult cattle infected with BLV develop persistent lymphocytosis, and only a small portion (0.5-10\%) of BLV-infected animals develops EBL [2]. Asymptomatic carrier of BLV-infected animals can be a source of infection. Therefore, EBL is a kind of hidden disease; Once EBL case was found, there may be many asymptomatic BLV- infected cattle around the case, which can spread silently without showing any clinical symptoms.

Historically, EBL was reported in 1871 in German medical literature. Early in the 20th century, it attracted attention in Eastern Europe, especially in Denmark and Germany, where a high incidence of a similar disease observed. EBL was supposed to be imported from Europe to USA at the end of 19th Century [3]. In Japan, the first EBL was described in 1926 in Iwate, a northern part of Japan. In 1969, BLV, the causative agent of EBL, was isolated in the USA from cattle with persistent lymphocytosis.

Retrovirus including BLV shows a unique replication cycle, i.e., the viral genome is integrated into the host chromosome. As a character of retrovirus infection, once the virus infects, the infection lasts as far as the host lives (life-long infection). Regarding the BLV transmission route, living lymphocytes carrying BLV are thought to be required for establishing new infection. Vertical infection including in uterus, birth canal and colostrum from BLV infected mother is known. Horizontal transmission includes iatrogenic/artificial contaminated blood exposure, direct contact and blood-siphoning insects. Mechanical transmission of BLV-infected lymphocytes by blood-siphoning insects such as biting flies are known. The incidence increase in summer or fall when those insects become active. Higher incidence in warmer climate and in wetter areas is also known. BLV-infected lymphocytes on the mouth-pad of insects can be transferred into the new cattle.

Although EBL is widely distributed in the world, eradication was succeeded in some countries, where test and cull policy was conducted. High prevalence of BLV infection is reported in Japan as well as USA. However, the prevalence of BLVinfection is not known regarding the countries where no surveillance was conducted.

In Japan, Bovine Leukosis (BL) became reportable disease in the year of 1998, because the government noticed the increased number of EBL cases. BL includes EBL and sporadic BL (SBL). EBL is caused by BLV, but the etiology of SBL is unknown. More than $90 \%$ of BL cases in Japan is thought to be EBL, although the precise diagnosis may be difficult in some cases. Traditionally, EBL is observed in the cattle more than 3 years old, but recently young animal below 2 years old are sometimes diagnosed as EBL [4]. 
Nationwide surveillance in Japan reported that seropositivity of BLV in dairy cattle and beef cattle increased from $7 \%$ and $11 \%$ in 1980 , to $41 \%$ and $29 \%$ in 2010 , respectively [5]. It also increased by age, from $20 \%$ at 0 year to $45 \%$ at $4-6$ years in dairy cattle, and from $14 \%$ at 0 year to $20 \%$ at 3-10 years in beef cattle, suggesting that the horizontal transmission plays a role.

Economic loss caused by BLV infection is not well understood, because most of the cases are asymptomatic. It is obvious that the fatal EBL results in loss of whole cattle. Among the EBL cases detected in the slaughterhouse, only 3\% of the cases showed symptom, and the rest of $97 \%$ cases was found by the investigation at the slaughterhouse. In addition to EBL, it is suggested that the persistent infection of BLV lead to the immune suppression, which may be associated with the infection of other pathogens and ineffectiveness of vaccination. Possible loss by BLV-infection includes milk production and survival, although there are some controversial reports.

In order to control EBL, understanding the status of BLVinfected cattle is critical. For diagnosis, BLV-specific-antibody in serum can be detected by ELISA. Viral load in peripheral blood can be quantified by real time PCR against BLV.

In 2015, Ministry of Agriculture, Fishery and Forestry (MAFF) in Japan released a guideline for controlling EBL [6]. This guideline describes the risk of transmission including iatrogenic/artificial infection of BLV. NOSAI, the nationwide Livestock Insurance based on the framework of Japan's Agricultural Scheme, started compensating EBL loss, as far as the farmers follow the guideline. This compensation system is expected to help raising awareness of BLV control measure to the farmers.

For management, eradication may be achieved by test and cull/slaughter, if the BLV positivity is low. If not, test and segregation is preferred not to spread BLV to the other animals [7-8]. Freezing treatment on colostrum from BLV positive cattle is effective to prevent the infection by destroying the BLVinfected lymphocytes [9]. Fly-net and insecticide are useful for preventing vector-bone infection.

Suitable management of BLV-infection for the region is to be carried out for sustainable development of livestock industries. Not only acute diseases, but also this kind of slow diseases needs to be concerned for better livestock production.

\section{REFERENCES}

[1] FAO, http://www:fao.org/docrep/014/i2373e/i2373e.pdf.

[2] Enzootic Bovine Leukosis. OIE Terrestrial Manual, 2012. Chapter 2.4.10: p. 721-732.

[3] Maclachlan, J.a.D., ED ed, Bovine Leukemia Virus in Chapter 14Retroviridae. Fenner's Veterinary Virology (Fourth Edition), 2011: p. 264266.

[4] Oguma, K., M. Suzuki, and H. Sentsui, Enzootic bovine leukosis in a twomonth-old calf. Virus Res, 2017. 233: p. 120-124.

[5] Murakami, K., et al., Nationwide survey of bovine leukemia virus infection among dairy and beef breeding cattle in Japan from 2009-2011. J Vet Med Sci, 2013. 75(8): p. 1123-6.

[6] Guideline for Enzootic Bovine Leukosis. Ministry of Agriculture, Forestry and Fisheries, Japan, 2015.

[7] Kobayashi, S., et al., The role of neighboring infected cattle in bovine leukemia virus transmission risk. J Vet Med Sci, 2015. 77(7): p. 861-3.

[8] Nekouei, O., et al., Herd-level risk factors for infection with bovine leukemia virus in Canadian dairy herds. Prev Vet Med, 2015. 119(3-4): p. 105-13.

[9] Kanno, T., et al., Effect of freezing treatment on colostrum to prevent the transmission of bovine leukemia virus. J Vet Med Sci, 2014. 76(2): p. 2557. 